Research Article

\title{
On Zero-Inflated Hierarchical Poisson Models with Application to Maternal Mortality Data
}

\author{
Kassim Tawiah $\mathbb{D}^{1},{ }^{1}$ Samuel Iddi $\mathbb{D}{ }^{2}$ and Anani Lotsi ${ }^{2}$ \\ ${ }^{1}$ Department of Mathematics and Statistics, University of Energy and Natural Resources, Sunyani, Ghana \\ ${ }^{2}$ Departments of Statistics and Actuarial Sciences, University of Ghana, Accra, Ghana \\ Correspondence should be addressed to Kassim Tawiah; kassim.tawiah@uenr.edu.gh and Samuel Iddi; siddi@ug.edu.gh
}

Received 11 May 2020; Revised 4 September 2020; Accepted 7 December 2020; Published 28 December 2020

Academic Editor: Harvinder S. Sidhu

Copyright $(92020$ Kassim Tawiah et al. This is an open access article distributed under the Creative Commons Attribution License, which permits unrestricted use, distribution, and reproduction in any medium, provided the original work is properly cited.

Count outcomes are commonly encountered in health sector data. The occurrence of count outcomes that exhibit many zeros has necessitated the extension of the ubiquitous Poisson regression model to accommodate the zero inflation and overdispersion as a result of the extra dispersion. We explored different extensions of the Poisson model including mixed models within the generalized linear mixed model framework to account for the repeated measurement of outcomes. These models are applied to maternal mortality data from fifty-six health facilities in four regions of Ghana. The objective is to identify factors associated with maternal mortality. The best-fitting model, the zero-inflated Poisson generalized linear mixed model, revealed that maternal mortality in hospital facilities is influenced by the number of referrals (into and out) of the hospital facility, number of antenatal visits exceeding four, number of midwives, and number of medical doctors at the facility. To be able to achieve targeted results in reducing maternal mortality and achieve the Sustainable Development Goal 3, the government, together with the ministry of health, should provide adequate maternal health services, especially at the district and community level. Additionally, there is a need for increased investment in Community Health Planning Services and related healthcare infrastructure and systems within the context of the Ouagadougou Declaration, that is, improve the training of skilled birth workers (midwives and doctors) and employ them at clinics to deal with labour complications without referring them to major hospitals. Furthermore, a wellstructured awareness campaign is needed with importance given to avoiding adolescent pregnancy and improving antenatal care attendance to, at least, four, the gold standard, before delivery. Also, we recommend quality assessment form an essential part of all services that are directed towards improving maternal health and that more emphasis is needed to be given on research with multiple allied partners.

\section{Introduction}

Most data in the health sector are based on counts. As such, the Poisson regression model, the simplest form of the generalized linear model for count outcome and a member of the exponential family $[1,2]$, provides a suitable modelling approach. Very commonly, these types of count outcomes in the health sector (especially when the event is rare) have an excessive number of zeros relative to the Poisson distribution. This renders the variance of the Poisson distribution to be far greater than the mean (mean equal to variance in a Poisson distribution), a phenomenon commonly referred to as overdispersion. Thus, the Poisson model has been extended to accommodate the excess number of zero counts and the remaining sources of heterogeneity causing overdispersion. For longitudinal data, the extension is necessary to correct the hierarchical structures and the possible correlation within outcomes from the same subject.

The Poisson distribution was extended to accommodate excess zeros by mixing a discrete mass and the Poisson distribution to obtain the zero-inflated Poisson model [3]. The discrete mass, usually a binomial distribution, is assumed to generate only the zeros, whereas the Poisson distribution generates both zeros and counts.

There has been tremendous work on zero-inflated models. Hall [4] modelled a zero-inflated Poisson with additional subject-specific random effects (only on the Poisson part). Lee et al. [5] used an independent random 
effect for both the Poisson and the binary mixture components. It is, therefore, interesting to consider the Poisson regression model with independent, correlated, or shared random effects and study the features of both components of the zero-inflated Poisson models.

Zhu et al. [6] extended the zero-inflated models to account for random effects heterogeneity by modelling their variance as a function of covariates. They envisaged through simulation that ignoring intervention and covariate-specific heterogeneity can produce biased estimates of covariate and random effects. Zhu et al. [6] proposed that biased estimates can be rectified by correctly modelling the random effects.

Xie et al. [7] developed a score test for homogeneity of the dispersion parameter in zero-inflated Poisson mixed regression models. They also determined the corresponding test statistic. However, they only probed the sampling distribution and power of the score test statistic through Monte Carlo simulation.

A recent study by Ghasemi et al. [8] emphasized the application of correlated count data in health and medical studies. They introduced double-inflated Poisson models for zero-inflated and count-inflated data.

Maternal mortality ratios are a function of both economic and social development of a country and the one that distinguishes between developed and developing nations. Reducing maternal mortality is not just an issue of development, but an issue of human rights [9]. Ghana missed the Millennium Development Goals (MDG) [10] and so signed on to the Sustainable Development Goal (SDG) [11], particularly in the area of health. A lot of efforts have been made by successive governments to reduce the menace, and this paper is an effort to generate evidence to support policymaking.

Various interventions introduced by the government through the Ghana Health Service to improve maternal healthcare include free maternal health services, repositioning family planning and training, as well as repositioning reproductive and child health staff; safe motherhood task force and increased production of midwives and doctors; and implementation of the High-Impact Rapid Delivery (HIRD). Others include the Ghana VAST Survival Programme; Prevention of Maternal Mortality Programme (PMMP); Safe-Motherhood Initiative; Making Pregnancy Safer Initiative; Prevention and Management of Safe Abortion Programme, Maternal and Neonatal Health Programme, Roll-Back Malaria Programme; and Intermittent Preventive Treatment (IPT) and Emergency Obstetric and Neonatal Care (EmONC) in all ten regions. Despite all these, several challenges and bottlenecks have been identified in maternal health services: inadequate maternal health services, especially at the district level, as well as investment in Community Health Planning Services and related Primary Healthcare infrastructure and systems within the context of the Ouagadougou Declaration. Improving the development of skilled health workers (midwives and doctors), the supply of equipment, logistics, staff accommodation, transportation, and ambulance services in addressing human resource constraints and poor-quality healthcare continue. Referrals remain a problem in many districts although teaching, regional, and district hospitals are well equipped to handle complicated labour cases. The main issue is how to timely transport women in labour to these facilities. Since the National Health Insurance Scheme (NHIS) does not cover the cost of conveying women in labour to these facilities, they often feel reluctant to be transported to the hospitals. Also, the unavailability of data and rigorous statistical interrogations on maternal healthcare for a systematic investigation into maternal health and lack of wellstructured plans and procedures to check and access where maternal programmes are absent vis-à-vis governmental attention are also major challenges [12].

Rai et al. [13] emphasized the need to acknowledge the social correlates of maternal deaths. Investigating and in-depth understanding of each maternal death can provide indications on practical ways of addressing the problem. The death of a mother has serious implications for the child, as well as other family members, and to prevent the same, a comprehensive approach is required. This could include providing essential maternal care, early management of complications, and goodquality intrapartum care through the involvement of skilled birth attendants. Ensuring the availability, affordability, and accessibility of quality maternal health services, including emergency obstetric care (EmOC), would prove pivotal in reducing maternal death. They were of the view that, to increase the perceived seriousness of the community regarding maternal health, a well-structured awareness campaign is needed with importance given to avoiding adolescent pregnancy. Also, quality assessment should form an essential part of all services that are directed towards improving maternal health, and more emphasis is needed to be given on research by involving multiple allied partners, to develop a prioritized, coordinated, and innovative research agenda for women's health.

Most studies on maternal mortality in Ghana are based on a single health facility or a single district or few districts [14-17]. Gumenga et al. [17] studied the maternal mortality pattern at the Tamale Teaching Hospital, while Asamoah et al. [15] observed the cause-specific maternal deaths among different socioeconomic groups in Ghana. Der et al. [16] noted that, at the Korle-Bu Teaching Hospital, 517 of the 634 pregnancy-related deaths $(81.5 \%)$ occurred in the community or within 24 hours of admission to the health facility with 117 (18.5\%) occurring at the health facility. Apanga and Awoonor-Williams [14] were of the view that lack of logistics, medical, and laboratory equipment and inadequate knowledge about the benefits of antenatal care services, as well as nonadherence of health workers to treatment protocols and standard operating procedures, were a major setback to the effective provision of maternal healthcare services in the Upper East region of Ghana. In this study, we used a longitudinal approach to identify factors that contribute to maternal mortality in fifty-six health facilities, randomly selected from four regions in Ghana, using data from 2010 to 2013.

\section{Data and Methods}

2.1. Data. The data used in this paper are obtained from health facilities in the Central, Eastern, Western, and Greater 
Accra regions spanning the years from 2010 to 2013. The health facilities (HFs) were made up of three (3) teaching hospitals (TH), two (2) regional hospitals (RH), twentytwo(22) general hospitals (GH), three (3) polyclinics (PC), eighteen (18) health centers (HC), three (3) clinics (CI), and five (5) community health planning services (CHIPS) compound.

The response variable was the number of maternal deaths at the health facility. The explanatory variables included the type of HF, location of the HF, existence of an emergency obstetric care (EmOC), number of deliveries at the health facility, number of doctors at the HF, number of midwives at the HF, number of paramedical staff at the maternity ward, number of obstetric cases with HIV/AIDS, number of obstetric cases with malaria, number of obstetric referrals from HF, and number of referrals to HF.

To obtain our data, a letter was written to the regional health directorates in all the regions included for their approval. After their approval, a follow-up letter was sent to various health directorates and the head of the health facilities at the district, subdistrict, and community levels of all the health facilities to seek their consent for the use of these secondary data. The data were subsequently collected from the various biostatistics/records departments and maternity ward record books after approval at the district, subdistrict, and community levels of all the health facilities. We considered years before the end of the MDGs to provide a clear direction on the barriers that hindered the achievement of the MGD 5 in Ghana in order to assist policymakers on where to channel their efforts in their quest to achieve the SDG 3.

From Figure 1, it can be seen that the data contained 187 zero counts making up $83.48 \%$ of the response variable which is the evidence of zero inflation. The hospital facility profiles showed higher variability between clinics $(\mathrm{HC}, \mathrm{Cl}$, CHPS, and PC) and hospitals (GH, RH, and TH) as compared to within-hospital facilities, indicating possible correlated effects. The clinics accounted for a greater proportion of the zeros. This is because the clinics generally refer all labour complications to the hospitals since it is believed that the clinics are unable to provide essential maternal care, early management of complications, and good quality intrapartum care through the involvement of skilled birth attendants. Emergency Obstetric Care (EmOC) is completely unavailable at the clinics.

To obtain an appropriate model to describe the data, we began with the Poisson regression model and consider all possible extensions.

2.2. Methods. Suppose that $Y_{i}$ represents the maternal death count from hospital $i=1,2,3, \ldots, n$. The Poisson regression assumes that $Y_{i}$ follows a Poisson distribution with mean, $\mu_{i}$. The mean is related to the set of $p$ covariates, $\mathbf{X}_{i}$, through a $\log$-link function. That is, $E\left(Y_{i}\right)=\mu_{i}=\exp \left(\boldsymbol{\beta}^{\prime} \mathbf{X}\right)$, where $\boldsymbol{\beta}$ is the effect of the covariates. The variance of $Y_{i}$ is also $\mu_{i}$. In many applications with count data, the observed variance is higher than the mean, leading to overdispersion [18]. This strict mean-variance relationship makes the Poisson model overly restrictive and inappropriate when the data exhibits overdispersion.

When the count data are collected repeatedly over time or clustered, correlation is induced and the independent assumption of the above mentioned models is violated. To address this, random effects are introduced in the linear part of the relationship involving the marginal means. For example, in the Poisson model, $\log \left(\mu_{i j}\right)=\boldsymbol{\beta}^{\prime} \mathbf{X}+\mathbf{Z}^{\prime} \mathbf{b}$, where $\mu_{i j}$ is the marginal mean of subject, $i$, at time $j$ or cluster $j$, $\mathbf{b} \sim N(0, \mathbf{D})$ is a vector of random effects, and $Z$ is a set of predictors associated with the random effects. The likelihood of the model is obtained by integrating out the random effects. That is, $L=\int \prod_{i=1}^{n} f\left(Y \mid \mathbf{b}_{i}\right) d \mathbf{b}_{i}$. For the Poisson mixed-effects model with random intercepts only, the marginal mean and variance are, respectively,

$$
\begin{gathered}
E\left(Y_{i}\right)=\mu_{i}=\exp \left(\boldsymbol{\beta}^{\prime} \mathbf{X}+\frac{d^{2}}{2}\right), \\
\operatorname{var}\left(Y_{i}\right)=\mu_{i}\left(1+\mu_{i}\left(\exp \left(d^{2}\right)-1\right)\right),
\end{gathered}
$$

where $d^{2}$ is the variance of the random intercept. The likelihood of the Poisson mixed-effects model with random intercepts only is, thus, given by

$$
L=\int_{0}^{\infty} \prod_{i=1}^{n} \frac{\left[\exp \left(\boldsymbol{\beta}^{\prime} \mathbf{X}+\left(d^{2} / 2\right)\right)\right]^{y_{i}} \exp \left[-\exp \left(\boldsymbol{\beta}^{\prime} \mathbf{X}+\left(d^{2} / 2\right)\right)\right]}{y_{i} !} \frac{1}{\sqrt{2 \pi d^{2}}} \exp \left(-\frac{1}{2 d^{2}} b_{i}^{2}\right) \mathrm{d} b_{i}
$$

From these expressions, it is clear that the random effects allow the generalized mixed models to account for the overdispersion through the parameter, $d$. Both overdispersion and correlation can happen together, and this led Molenberghs et al. [19] to formulate a flexible and unified modelling framework, which they termed the combined model, to simultaneously capture overdispersion and correlation for a wide range of clustered data, including counts, binary, and time-to-event.

As maternal mortality occurs within hospital facilities, the referrals from one facility to another may imply that no deaths are reported within a particular time, hence triggering a phenomenon that appears in many health services; the excessive number of zero counts, more than expected, 


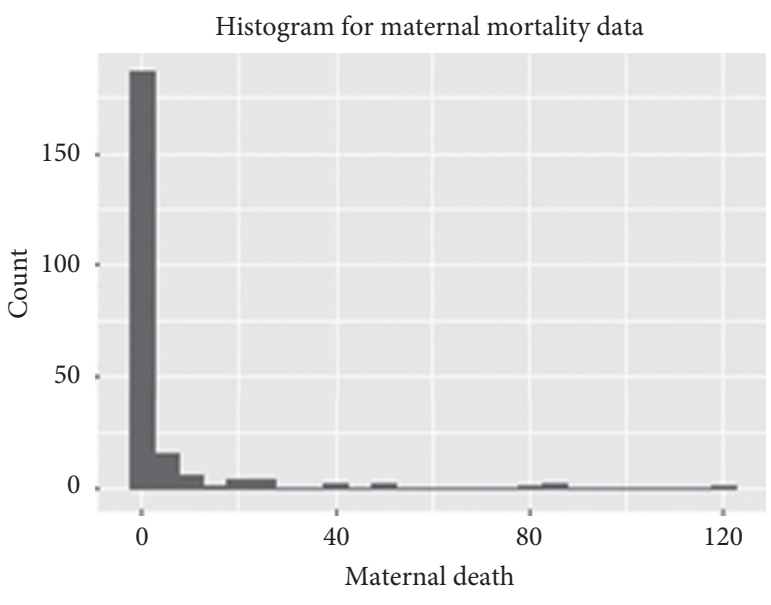

(a)

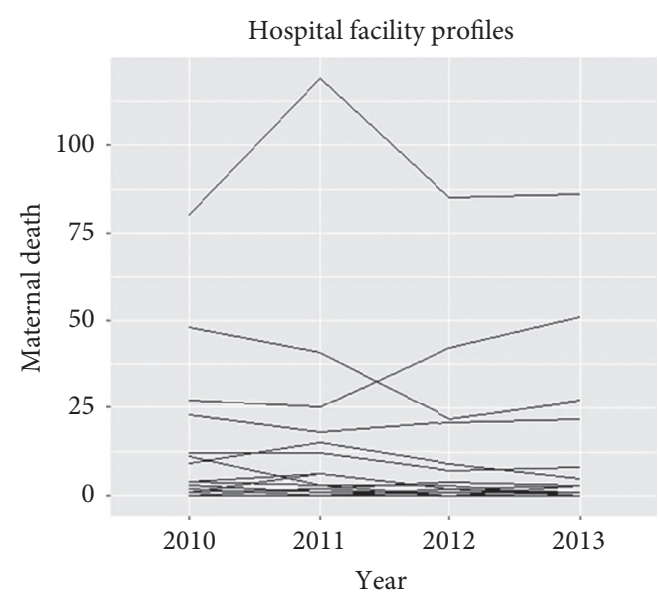

(b)

Figure 1: Maternal mortality data. Histogram and hospital facility profiles.

relative to a Poisson distribution. Such data are fitted as a zero-inflated model [3]. In a zero-inflated model, we assume the zeros come from two processes. The first process generates only zeros with probability, $\pi_{i}$ for observation $i$, and the second process generates counts with probability, $1-\pi_{i}$. Thus, for a zero-inflated model, the probability distribution is given by

$$
P\left(Y_{i}=y_{i}\right)= \begin{cases}\pi_{i}+\left(1-\pi_{i}\right) f_{i}\left(0 \mid \lambda_{i}\right), & \text { if } y_{i}=0 \\ \left(1-\pi_{i}\right) f_{i}\left(y_{i} \mid \lambda_{i}\right), & \text { if } y_{i}>0\end{cases}
$$

where $\pi_{i}$ and $\lambda_{i}$ are functions of covariates. Link functions, such as logit or probit, can be used to transform $\pi_{i}$, and the common log link is used for $\lambda_{i}$. For a zero-inflated Poisson generalized linear model (ZIP) the probability density function is given by

$$
P\left(Y_{i}=y_{i}\right)= \begin{cases}\pi_{i}+\left(1-\pi_{i}\right) \exp \left(-\lambda_{i}\right), & \text { if } y_{i}=0, \\ \left(1-\pi_{i}\right) \frac{\exp \left(-\lambda_{i}\right) \lambda_{i}^{y_{i}}}{y_{i} !}, & \text { if } y_{i}>0\end{cases}
$$

leading to $E\left(Y_{i}\right)=\left(1-\pi_{i}\right) \lambda_{i}$ and $\operatorname{var}\left(Y_{i}\right)=\lambda_{i}+\left(\pi_{i} / 1-\right.$ $\left.\pi_{i}\right) \lambda_{i}^{2}$, with $\log \left(\pi_{i} /\left(1-\pi_{i}\right)\right)=\boldsymbol{\alpha}^{\prime} \mathbf{Z}$ and $\log \left(\lambda_{i}\right)=\boldsymbol{\beta}^{\prime} \mathbf{X}$ where $\boldsymbol{\alpha}, \boldsymbol{\beta}$ are vectors of parameters with $\mathbf{Z}$ and $\mathbf{X}$, respectively.

The ZIP is not suitable for correlated data. They are further extended to ZI Poisson GLMM (ZIPG) to correct for dependency in the data. In this case, random effects are introduced as in the generalized mixed models discussed above in either the zero-inflated or Poisson part of the model or both parts. For the ZIPG with random intercepts only, the mean and variance are, respectively,

$$
\begin{gathered}
E\left(Y_{i}\right)=\exp \left(\boldsymbol{\beta}^{\prime} \mathbf{X}+\frac{d^{2}}{2}\right)\left[1+\exp \left(\boldsymbol{\alpha}^{\prime} \mathbf{Z}+\frac{d^{2}}{2}\right)\right]^{-1}, \\
\operatorname{var}\left(Y_{i}\right)=\exp \left(\boldsymbol{\beta}^{\prime} \mathbf{X}+\frac{d^{2}}{2}\right)\left(1+\exp \left(\boldsymbol{\beta}^{\prime} \mathbf{X}+\boldsymbol{\alpha}^{\prime} \mathbf{Z}+d^{2}\right)\right),
\end{gathered}
$$

where $\boldsymbol{\alpha}, \boldsymbol{\beta}$ are vectors of parameters with $\mathbf{Z}$ and $\mathbf{X}$, respectively, and $d^{2}$ is the variance of the random intercept. The likelihood of the ZIPG with random intercepts only can be expressed as

$$
\begin{aligned}
L= & \int_{0}^{\infty} \prod_{i=1}^{n} I\left(y_{i}>0\right)\left[\frac{\exp \left(\boldsymbol{\alpha}^{\prime} \mathbf{Z}+\left(d^{2} / 2\right)\right)}{1+\exp \left(\boldsymbol{\alpha}^{\prime} \mathbf{Z}+\left(d^{2} / 2\right)\right)}+\left(\frac{1}{\exp \left(\boldsymbol{\alpha}^{\prime} \mathbf{Z}+\left(d^{2} / 2\right)\right)}\right) \exp \left(-\exp \left(\boldsymbol{\beta}^{\prime} \mathbf{X}+\frac{d^{2}}{2}\right)\right)\right] \frac{1}{\sqrt{2 \pi d^{2}}} \exp \left(-\frac{1}{2 d^{2}} b_{i}^{2}\right) \mathrm{d} b_{i} \\
& \times \int_{0}^{\infty} \prod_{i=1}^{n} I\left(y_{i}>0\right)\left(\frac{1}{\exp \left(\boldsymbol{\alpha}^{\prime} \mathbf{Z}+\left(d^{2} / 2\right)\right)}\right) \frac{\left[\exp \left(\boldsymbol{\beta}^{\prime} \mathbf{X}+\left(d^{2} / 2\right)\right)\right]^{y_{i}} \exp \left[-\exp \left(\boldsymbol{\beta}^{\prime} \mathbf{X}+\left(d^{2} / 2\right)\right)\right]}{y_{i} !} \frac{1}{\sqrt{2 \pi d^{2}}} \exp \left(-\frac{1}{2 d^{2}} b_{i}^{2}\right) \mathrm{d} b_{i} .
\end{aligned}
$$

All parameters in the likelihoods, which are easy to formulate, as well as the standard errors of the ZIPG, are obtained using the Expectation-Maximization (EM) algorithm.
The model comparison is based on the Akaike Information Criterion (AIC; [20-22]) and the Bayesian Information Criterion (BIC; [23]). These metrics combine a measure of model fit, typically twice the negative log- 
likelihood, with a penalty for model complexity, expressed as a function of the number of parameters. Models with smaller AIC and BIC are preferable. The AIC and BIC are estimated, respectively, by the following formulas:

$$
\begin{aligned}
\text { AIC }= & 2 q-2 \log (\text { Likelihood })=2 q \\
& +n \log (\text { residual sum of squares }+n \log (n)), \\
\text { BIC }= & q \log (n)-2 \log (\text { Likelihood }) \\
= & \log (\text { error variance })+\frac{q}{n} \log (n),
\end{aligned}
$$

where $q$ is the number of parameters in the model and $n$ is the number of observations.

The models were fitted using the lme4, MASS, glmmTMB, and pscl packages in the $R$ statistical software using maximum likelihood estimations. The MASS package was used to fit the Poisson GLM (P), the lme4 for the Poisson GLMM (PG), the pscl for zero-inflated Poisson GLMs (ZIPG), and the $\operatorname{glmm} T M B$ for the zero-inflated Poisson GLMMs (ZIPG).

\section{Results and Discussions}

The mean and variance of the response were 4.01 and 203.78, respectively, indicating overdispersion. We began with the Poisson generalized linear regression model.

Using the stepwise deletion procedure in $R$, nonsignificant explanatory variables of the Poisson generalized linear regression were eliminated with the significance level set at 0.05 . The significant variables included year, region, number of antenatal visits exceeding four, number of referrals into the hospital facility, number of referrals out of the hospital facility, number of deliveries at the facility, number of obstetric cases with malaria, number of medical doctors, and number of midwives at the hospital facility. These variables were used in all model extensions. However, a stepwise deletion procedure was used at every stage to delete nonsignificant explanatory variables. The significant variables at each stage were used for the next.

The AIC and BIC were obtained for all extended models (Table 1). The values indicated that the Poisson generalized linear regression model performs poorly for zero-inflated and overdispersed data. The best model was the zero-inflated Poisson generalized linear mixed model (ZIPG) since it had the least value. The descriptive statistics of the response and explanatory variables in the model are presented in Table 2. Interestingly, the Poisson generalized linear mixed model (PG) did better than the zero-inflated Poisson generalized linear model (ZIP) in terms of AIC and BIC values. This may imply that the PG may have corrected for excess zeroes more than the ZIP.

When interpreting the effect of parameters in a zeroinflated model, an aversion for an explanatory variable is shown when the explanatory variable indicates an increasing coefficient in the zero process and a decreasing effect in the parent count process. However, an attraction is said to occur
TABle 1: Maternal mortality data. AIC and BIC comparison for all models

\begin{tabular}{lcc}
\hline Models & AIC & BIC \\
\hline P & 806.09 & 864.08 \\
ZIP & 714.56 & 738.08 \\
PG & 667.63 & 687.21 \\
ZIPG & 537.60 & 582.00 \\
\hline
\end{tabular}

when the zero part for the explanatory variable shows a negative coefficient implying a decreasing effect and a positive coefficient indicating an increasing effect in the parent count process [24].

Parameter estimates and their corresponding standard errors were obtained for the ZIPG (Table 3). For the conditional model, the intercept was 2.3553 with a $p$ value less than 0.001 . That is, the intercept is statistically significant at 0.05 . This means that the estimated expected maternal death count is 10.5413 when there are no doctors, no midwives, no referrals, no antenatal visits exceeding four, no expectant mother having HIV/AIDS, and no expectant mother having malaria. This could mean that the expected number of maternal death will be very high if expectant mothers use traditional birth attendants or deliver at home.

For referral in (when a particular health facility with better infrastructure and human resource admits expectant mothers in labour with complications from health facilities with less infrastructure and human resources to deal with the complications), the estimate is 0.0010 with a $p$ value less than 0.001 . Thus, the number of referrals into a hospital facility has a significant increasing effect on the expected number of maternal death. When all other explanatory variables are kept constant, a unit increase in the number of referral-in yields a count ratio of 1.0010 . This translates into a $0.10 \%$ increment in the expected number of maternal death if all other explanatory variables are kept constant.

Referral out (a situation whereby a particular health facility with less infrastructure and human resources transport expectant mothers in labour with complications to another facility with better infrastructure and human resources to deal with the complications) was significant with a count ratio of 0.9858. That is, the number of referrals out of a hospital facility has a decreasing effect on the expected number of maternal death. This means that a unit increase in the number of referrals out leads to a decrement of $1.42 \%$ in the expected number of maternal death. This is obvious as health facilities with a lot of referrals out will certainly have a decline in the number of maternal deaths or no maternal deaths.

Malaria and HIV/AIDS in pregnancy were both not significant. However, they both have a diminishing effect on the expected number of maternal death. Generally, pregnant women who are found to have malaria during their antenatal visits are directed to see doctors for proper attention and medication to help nullify the effect of the disease and cure them. Similarly, those found with HIV/AIDS are put on an essential medication by doctors to help them stay healthier. We can attribute this to the Roll-Back Malaria and HIV/ AIDS Campaign initiatives by the government and its stakeholders. 
Table 2: Maternal mortality data. Descriptive statistics of the response and explanatory variables.

\begin{tabular}{lccccc}
\hline Variable & No. of observations & Minimum & Maximum & Mean (SD) & Median (IQR) \\
\hline Maternal death & 224 & 0 & 119 & $4.01(14.28)$ & $0(0.00-0.25)$ \\
Referral in & 224 & 0 & 4716 & $118.40(596.39)$ & $0(0.00-7.00)$ \\
Referral out & 224 & 0 & 820 & $29.47(67.50)$ & $11(0.00-30.50)$ \\
Malaria & 224 & 0 & 750 & $106.39(146.01)$ & $48(7.75-144.75)$ \\
HIV/AIDS & 224 & 0 & 207 & $19.20(31.41)$ & $9(2.00-20.25)$ \\
Doctors & 224 & 0 & 73 & $2.57(9.37)$ & $0(0.00-2.25)$ \\
Midwives & 224 & 0 & 382 & $13.28(48.81)$ & $2(1.00-10.00)$ \\
ANC & 224 & 0 & 9619 & $826.90(1383.86)$ & $412(121.00-1044.80)$ \\
\hline
\end{tabular}

TABle 3: Maternal mortality data. Parameter estimates and standard errors (s.e.) for the zero-inflated Poisson GLMM (ZIPG).

\begin{tabular}{lcc}
\hline & Estimates (s.e.) & $\operatorname{Pr}(>|z|)$ \\
\hline Conditional model & ZIPG & \\
Intercept & $2.3553(0.3884)$ & $<0.001$ \\
Referral in & $0.0010(0.0003)$ & $<0.001$ \\
Referral out & $-0.0143(0.0030)$ & $<0.001$ \\
Malaria & $-0.0008(0.0005)$ & 0.150 \\
HIV/AIDS & $-0.0017(0.0022)$ & 0.434 \\
Doctors & $-0.7479(0.0192)$ & $<0.001$ \\
Midwives & $0.0978(0.0042)$ & $<0.001$ \\
ANC & $0.0015(0.0006)$ & $<0.001$ \\
\hline Zero-inflation model & & $<0.001$ \\
Intercept & $3.6729(0.5349)$ & 0.018 \\
Referral in & $-0.0472(0.0200)$ & 0.129 \\
Referral out & $0.0161(0.0106)$ & $<0.001$ \\
Midwives & $-0.2972(0.0570)$ & \\
\hline Random effects & & Variance \\
Groups & Name & 0.1279 \\
Year & Intercept & 0.4067 \\
Region & Intercept &
\end{tabular}

The number of doctors and midwives at a particular health facility were found to be statistically significant. The number of doctors at a hospital facility has a decreasing effect on the expected number of maternal death. A unit increase in the number of doctors, preferably gynecologists, diminishes the expected number of maternal death by $52.66 \%$ if all other explanatory variables remain constant. This implies that a unit increase in the number of doctors present during delivery can reduce the number of maternal deaths significantly. This is because doctors are the only ones who can help treat labour complications when they arise during delivery. Similarly, a unit increase in the number of midwives during delivery increases the expected number of maternal death by $8.12 \%$. Although midwives play a major role for women in labour, this could mean that they are unable to treat complications when they arise during delivery.

Antennal visits exceeding four (ANC) was significant. A unit increase in the number of antenatal visits in excess of four increases the expected number of maternal death by $0.15 \%$ if all other variables are kept constant. Even if a pregnant woman attends antenatal in excess of four, there is a slim chance of her dying during delivery. We could attribute this to the fact that most pregnant women attended antenatal at clinics where there are less infrastructure and human resources to discover any anomalies in the pregnancy.

Turning to the zero-inflation model, the intercepts was 3.6729 and significant at 0.05 . The number of referral-in and the number of midwives both had a significant effect on the odds of the expected number of maternal death. They both had a decreasing effect on the odds of the expected number of maternal death. Referral-out had no significant effect on the odds of the expected number of maternal death. Keeping other explanatory variables constant, the odds of the expected number of maternal death diminishes by $4.61 \%$ for a unit increase in referral in. The number of midwives at a hospital facility abates the odds of the number of maternal death by $25.71 \%$ for any unit increase. Referral out augments the odds of maternal death by $1.62 \%$ for any unit increase. We observed attraction for referral in and midwives, but aversion for referral out.

\section{Conclusions}

In the presence of zero inflation and overdispersion, the poisson generalized linear regression model performed poorly.

This study explored different extensions of the Poisson model based on mixed models. Models were applied to maternal mortality data from fifty-six health facilities in four regions of Ghana.

The overall best model, the zero-inflated Poisson generalized linear mixed model, revealed that maternal mortality in hospital facilities is influenced by the number of referrals (into and out) of the hospital facility, number of antenatal visits exceeding four, number of midwives, and the number of medical doctors at the facility which is similar to findings of Loquiha et al. [25]. We recommend that to be able to achieve targeted results in reducing maternal mortality and achieve the Sustainable Development Goal 3, the government, together with the ministry of health, should provide adequate maternal health services, especially at the district and community level. Additionally, there is a need for increased investment in Community Health Planning Services and related healthcare infrastructure and systems within the context of the Ouagadougou Declaration, that is, improve the training of skilled birth workers (midwives and doctors) and employ them at clinics to deal with labour complications without referring them to major hospitals. Furthermore, a well-structured awareness campaign is 
needed with importance given to avoiding adolescent pregnancy and improving antenatal care attendance to, at least, four, the gold standard, before delivery. Also, we recommend quality assessment form an essential part of all services that are directed towards improving maternal health and that more emphasis is needed to be given on research with multiple allied partners.

\section{Data Availability}

We used data on maternal mortality from 2010 to 2013 in health facilities in four regions of Ghana with permission from the Ghana Health Service. The data is the sole property of the Ghana Health Service. Data can be made available upon request from the Ghana Health service.

\section{Conflicts of Interest}

The authors declare no conflicts of interest.

\section{Authors' Contributions}

KT, SI, and AL conceived the idea and planned the study. KT collected the data. KT and SI performed the analysis. SI and AL suggested the statistical methodology and supervised the entire study. KT and SI wrote the manuscript. All authors agreed to be accountable for all aspects of the work and jointly own the work. All authors read and approved the final paper.

\section{Acknowledgments}

Special thanks are due to the University of Ghana-Carnegie Cooperation Next Generation of Academics in Africa Project whose financial support made the paper a viable one. The authors are grateful to all Regional and District Health Directors in Central, Eastern, Western, and Greater-Accra regions for their immense support. The authors thank the administrators, senior medical supervisors, and heads of the biostatistics/records department of all the health facilities for giving them the secondary data for the study. This study was fully funded by the University of Ghana-Carnegie Cooperation Next Generation of Academics in Africa Project.

\section{References}

[1] A. Agresti, Categorical Data Analysis, Wiley \& Sons, Inc., New York, NJ, USA, 2nd edition, 2002.

[2] J. A. Nelder and R. W. M. Wedderburn, "Generalized linear models," Journal of the Royal Statistical Society. Series A (General), vol. 135, no. 3, pp. 370-384, 1972.

[3] D. Lambert, "Zero-inflated Poisson regression, with an application to defects in manufacturing flated Poisson regression, with an application to defects in manufacturing," Technometrics, vol. 34, no. 1, pp. 1-13, 1992.

[4] D. B. Hall, "Zero-inflated Poisson and binomial regression with random effects: a case study," Biometrics, vol. 56, no. 4 , pp. 1030-1039, 2000.

[5] A. H. Lee, K. Wang, J. A. Scott, K. K. W. Yau, and G. J. McLachlan, "Multi-level zero-inflated Poisson regression modelling of correlated count data with excess zeros flated
Poisson regression modeling of correlated count data with excess zeros," Statistical Methods in Medical Research, vol. 15, no. 1, pp. 47-61, 2006.

[6] H. Zhu, S. Luo, and S. M. DeSantis, "Zero-inflated count models for longitudinal measurements with heterogeneous random effects," Statistical Methods in Medical Research, vol. 26, no. 4, pp. 1774-1786, 2017.

[7] F. C. Xie, J. G. Lin, and B. C. Wei, "Score test for homogeneity of dispersion in generalized Poisson mixed models with excess zeros," Communications in Statistics-Simulation and Computation, vol. 46, no. 1, pp. 301-314, 2017.

[8] E. Ghasemi, A. A. Baghban, F. Zayeri, A. Pourhoseingholi, and S. M. Safavi, "A double-inflated Poisson regression for correlated count data," Journal of Applied Statistics, pp. 1-7, 2020.

[9] P. Hunt and J. B. De Mesquita, Reducing Maternal Mortality: The Contribution of the Right to the Highest Attainable Standard of Health, University of Essex, Human Right Centre, UNFPA, Colchester, UK, 2012, https://www.unfpa.org/sites/ default/files/pub-pdf/reducing_mm.pdf.

[10] United Nations Millennium Development Goals, Report 2012, United Nations Millennium Development Goals, New York, NY, USA, 2012.

[11] United Nations Sustainable Development Goals, Sustainable Development Goals, United Nations Sustainable Development Goals, New York, NY, USA, 2015.

[12] Ghana Health Service, Maternal Health, Ghana Health Service, Accra, Ghana, 2014, https://www.ghanahealthservice. org/maternal-health.php.

[13] S. K. Rai, K. Anand, P. Misra, S. Kant, and R. P. Upadhyay, "Public health approach to address maternal mortality," Indian Journal of Public Health, vol. 56, no. 3, pp. 196-203, 2020.

[14] P. A. Apanga and J. K. Awoonor-Williams, "Maternal death in rural Ghana: a case study in the Upper East region of Ghana," Frontiers in Public Health, vol. 16, p. 101, 2018.

[15] B. O. Asamoah, K. M. Moussa, M. Stafstrom, and G. Musinguzi, "Distribution of causes of maternal mortality among different socio-demographic groups in Ghana; a descriptive study," BMC Public Health, vol. 11, pp. 159-168, 2011.

[16] E. M. Der, C. Moyer, R. K. Gyasi et al., "Pregnancy related causes of deaths in Ghana: a 5-year retrospective study," Ghana Medical Journal, vol. 47, no. 4, pp. 158-163, 2013.

[17] S. K. Gumanga, D. Z. Kolbila, B. B. N. Gandau, A. Munkaila, H. Malechi, and K. Kyei-Aboagye, "Trends in maternal mortality in Tamale teaching hospital," Ghana Medical Journal, vol. 45, no. 3, pp. 105-110, 2011.

[18] Y. Min and A. Agresti, "Random effect models for repeated measures of zero-inflated count data," Statistical Modelling: An International Journal, vol. 5, no. 1, pp. 1-19, 2005.

[19] G. Molenberghs, G. Verbeke, C. G. B. Demétrio, and A. M. C. Vieira, "A family of generalized linear models for repeated measures with normal and conjugate random effects," Statistical Science, vol. 25, no. 3, pp. 325-347, 2010.

[20] H. Akaike, "Information theory and an extension of the maximum likelihood principle," Proceedings of the Second International Symposium Theory, pp. 267-281, Springer, Berlin, Germany, 1973.

[21] H. Akaike, "A new look at the statistical model identification," IEEE Transactions on Automatic Control, vol. 19, no. 6, pp. 716-723, 1974.

[22] F. Vaida and S. Blanchard, "Conditional Akaike information for mixed-effects models," Biometrika, vol. 92, no. 2, pp. 351-370, 2005. 
[23] K. P. Burnham and D. R. Anderson, "Multimodel inference," Sociological Methods \& Research, vol. 33, no. 2, pp. 261-304, 2004.

[24] E. Somua-Wiafe, A. Asare-Kumi, E. N. N. Nortey, and S. Iddi, "Modelling vehicular crash mortalities in Ghana," Model Assisted Statistics and Applications, vol. 13, no. 3, pp. 387-295, 2018.

[25] O. Loquiha, N. Hens, L. Chavane, M. Temmerman, and M. Aerts, "Modeling heterogeneity for count data: a study of maternal mortality in health facilities in Mozambique," Biometrical Journal, vol. 55, no. 5, pp. 647-660, 2013. 